\title{
Pseudomonas aeruginosa isolates and their antimicrobial susceptibility pattern among catheterized patients at Jimma University Teaching Hospital, Jimma, Ethiopia
}

\author{
Temesgen Bekele ${ }^{1}$, Amene Tesfaye ${ }^{1}$, Tsegaye Sewunet ${ }^{2}$ and Habtewold Deti Waktola ${ }^{1 *}$
}

\begin{abstract}
Background: Pseudomonas aeruginosa is among the most common bacterial pathogens with wide spread distribution in health care settings. Despite advances in medical and surgical care and introduction of wide variety of antimicrobial agents, Pseudomonas aeruginosa continues to cause life threatening infection. Thus, this study aims to isolate and determine antimicrobial susceptibility patterns of Pseudomonas aeruginosa from catheterized patients with urinary tract infection.

Result: A cross-sectional study was conducted from January to May, 2013. Urine specimens of 73 catheterized patients who developed urinary tract infection after catheterization were collected from sampling port of the catheter. The urine samples were inoculated on MaConckey and blood agar plates, and incubated at $37^{\circ} \mathrm{C}$ for $24 \mathrm{~h}$. The isolates were identified by conventional microbiological tests. Antimicrobial susceptibility pattern was determined by modified Kirby-Bauer disk diffusion method. From a total of 73 urine samples collected P. aeruginosa was isolated from 36 (49.32\%) catheterized patients; 17 (23.29\%) males and 19 (26.03\%) females. While all P. aeruginosa isolates were found to be susceptible to Norfloxacin and Ciprofloxacin most isolates were also susceptible to Gentamicin (86.12\%).

Conclusion: The result shows higher prevalence of $P$. aeruginosa isolates among catheterized patients and the isolates were susceptible to the antimicrobials studied. All P. aeruginosa isolates were susceptible to Ciprofloxacin and Norfloxacin with some of the isolates shown resistance to Gentamicin. While the susceptibility of the isolates to the two fluoroquinolones is a good news for the prescribers their future rational prescription and use should be the main focus.
\end{abstract}

Keywords: Pseudomonas aeruginosa, Urinary tract infection, Clinical isolates, Antimicrobial susceptibility

\section{Background}

Pseudomonas aeruginosa is the third leading cause of hospital-acquired urinary tract infections (UTIs), accounting for about $12 \%$ of all hospital acquired infections. It can invade the blood stream from the urinary tract, and this has been shown to be the source of nearly $40 \%$ of Pseudomonas bacteremia. Urinary tract infection caused by $P$. aeruginosa are usually hospital-acquired and

\footnotetext{
*Correspondence: habtewold.deti@ju.edu.et

${ }^{1}$ Department of Pharmacy, College of Health Sciences, Jimma University,

P. O. Box: 378, Jimma, Ethiopia

Full list of author information is available at the end of the article
}

related to urinary tract catheterization instrumentation or surgery [1].

Infections caused by Pseudomonas aeruginosa are increasing both in hospital and in general community and it has been reported as one of the principal cause of nosocomial pathogen, particularly among immune compromised patient [1]. Concurrently, the extensive use of antimicrobial agents and the evolutionary antimicrobial resistance strategies of bacteria have resulted in the emergence of drug resistant bacteria. The efficiency of many antibiotics for treatment of infections has become quite limited due to the development of resistance and 
threat from antimicrobial resistant organisms is accumulating and accelerating [2].

P.aeruginosa was the second most common cause of pneumonia, the fourth most cause of urinary tract infection, and the sixth most common blood stream isolate in intensive care units (ICUs). Many potential reservoirs of infection have been identified in hospital environment, including respiratory equipment, cleaning solutions, disinfectants, sinks, vegetables, flowers, endoscopes, and physiotherapy pools [3].

The increasing prevalence of health-care associated infections (HAIs) produced by multidrug-resistant (MDR) P. aeruginosa strains severely compromises the selection of appropriate treatments and is therefore associated with significant morbidity and mortality [4]. Infections caused by $P$. aeruginosa are difficult to cure and often require combination therapy. For $P$. aeruginosa, antibiotic resistance is an increasing problem [5]. A varying degree of resistance to all known antipseudomonal antibiotics have been reported in different areas of the world by different authors [6-8]. Moreover, high rates of resistance to antibiotics are associated with nosocomial $P$. aeruginosa strains. It has been associated with sporadic or clustered cases of infection generally confined to single hospitalization units [8]. P. aeruginosa has been identified as the fourth most common causing catheterassociated urinary tract infections. The current increase in incidence of MDR isolates of $P$. aeruginosa raises serious concerns. Multidrug-resistance in $P$. aeruginosa is defined as the resistance to $\geq 3$ of the following classes of antibiotics: penicillin's/cephalosporin's/monobactams, carbapenems, amino glycosides, and fluoroquinolones [9].

An alarming increase in the prevalence of MDR P. aeruginosa from 36 to $52 \%$ were reported in an Egyptian study $[3,10]$. In studies done in Pakistan and Iran, 29 and $30 \%$ MDR phenotype was reported respectively [11].

\section{Methods}

A cross-sectional study was conducted at Jimma University Teaching Hospital from Jan to May 2013. All catheterized patients found during the study period were conveniently enrolled in the study. The laboratory tests were performed at teaching laboratory of medical microbiology at Department of Laboratory Sciences and Pathology.

\section{Specimen collection, isolation and identification}

Three to five milliliters of urine samples were collected through a sampling port (created just above where the storage bag tube fitted) from the catheter into clean and dry containers using 24 guage needle and syringe after cleaning the sampling port area with $70 \%$ alcohol [12].
The specimens were transported immediately to the lab and inoculated on MaConckey and blood agar, and incubated aerobically at $37^{\circ} \mathrm{C}$ for $24 \mathrm{~h}$. Non-lactose fermenting pale colonies from MaConckey and large flat dark greenish colonies from blood agar (after sub-culturing on nutrient agar) were tested for conventional biochemical tests: oxidase test, catalase, citrate utilization, and oxidative fermentation. The isolates were further sub-cultured on to two nutrient agar plates and incubated separately (at $37{ }^{\circ} \mathrm{C}$ for pigment production and growth at $42{ }^{\circ} \mathrm{C}$ ). Pseudomonas aeruginosa ATCC 27853 was used as a quality control strain.

Based on colony morphology, gram negative rod, oxidase positive, urease positive, Simon-citrates positive, and growth at $42{ }^{\circ} \mathrm{C}$ was confirmed as $P$. aeruginosa.

\section{Susceptibility tests}

Antimicrobial susceptibility tests were done by the Kirby-Bauer disk diffusion method as per the recommendations of [14] against a panel of antipseudomonal antimicrobials of standard strengths. From each isolated pure colony inoculums were prepared in physiological saline by adjusting the turbidity of bacterial suspension to 0.5 McFarland's standard; which is visually comparable to a microbial suspension of approximately $1.5 \times 10^{8} \mathrm{cfu} /$ $\mathrm{ml}$. Optimally, within $15 \mathrm{~min}$ after adjusting the turbidity of the inoculums suspension, a sterile cotton swab was dipped into the adjusted suspension. A dried surfaces of a Mueller-Hinton (Oxoid) agar plate were inoculated by spreading the swab over the entire sterile agar surface. This procedure is repeated by spreading two more times, rotating the plate approximately $60^{\circ}$ each time to ensure an even distribution of inoculums [13]. Then the rim of the agar is swabbed. The following antibiotic discs were tested: Ciprofloxacin $(5 \mu \mathrm{g})$, Gentamicin $(10 \mu \mathrm{g})$, and Norfloxacin $(10 \mu \mathrm{g})$ (Oxoid, Ltd., Basing stoke, Hampshire, England) standard antibiotic discs were placed on Muller-Hinton agar (Oxoid), which were previously inoculated with test strains and incubated at $37^{\circ} \mathrm{C}$ for 16-18 h. After incubation, inhibition zones were recorded as the diameter of the clear zones around the disc and interpreted according to performance standard for antimicrobial disk susceptibility test (CLSI 2012) [14].

\section{Results}

\section{Patients and specimens data}

Of the total 73 catheterized patients recruited in this study $51(69.68 \%)$ were male and $22(30.42 \%)$ were females. Urine specimen of these patients were examined using standard laboratory procedure and a total of $36(49.32 \%)$ cultures were found positive for $P$. aeruginosa. Of these positive cultures for P. aeruginosa, 19 $(26.03 \%)$ and $17(23.29 \%)$ were identified from urine 
Table 1 Distribution of Pseudomonas aeruginosa isolates among males and females

\begin{tabular}{lllc}
\hline Sex & No. tested (\%) & No. positive (\%) & No. negative (\%) \\
\hline Male & $51(69.86)$ & $17(33.33)$ & $34(66.67)$ \\
Female & $22(30.14)$ & $19(86.36)$ & $3(13.64)$ \\
Total & $73(100)$ & $36(49.32)$ & $37(50.68)$ \\
\hline
\end{tabular}

\section{Table 2 Distribution of Pseudomonas aeruginosa among different age groups}

\begin{tabular}{lll}
\hline Age group (years) & Number of isolates & Percentage (\%) \\
\hline $14-25$ & 4 & 11.12 \\
$26-35$ & 3 & 8.33 \\
$36-45$ & 8 & 22.32 \\
$>45$ & 21 & 58.33 \\
Total & 36 & 100
\end{tabular}

Table 3 Antimicrobial susceptibility patterns of Pseudomonas aeruginosa isolates

\begin{tabular}{llll}
\hline Antibiotics & \multicolumn{2}{l}{ AST profile } & \\
\cline { 2 - 4 } & Resistant (\%) & Intermediate (\%) & Susceptible (\%) \\
\hline Ciprofloxacin & - & - & $36(100)$ \\
Gentamicin & - & $5(13.88)$ & $31(86.12)$ \\
Norfloxacin & - & - & $36(100)$ \\
\hline
\end{tabular}

specimens of females and males respectively (Table 1). On the other hand; the proportion of positive cultures is very high among females (86.36) as compared to males (33.3). Most of the strains were isolated from older patients ( $\geq 45$ years) $(58.33 \%$ ) followed by $36-45$ years old patients (22.32\%) (Table 2).

\section{Antimicrobial susceptibility patterns}

Antimicrobial susceptibility patterns of $P$. aeruginosa to the three drugs commonly used in our setup was examined. While all $P$. aeruginosa strains were susceptible to Norfloxacin and Ciprofloxacin most strains were also susceptible for Gentamicin (86.12\%) (Table 3).

\section{Discussion}

In spite of advances in medical care and infection prevention policies and practices; Catheter associated urinary tract infections (CAUTIs) remains a major problem. The increasing number of CAUTIs bears on the fact that urinary catheters became second most often used foreign body inserted into human body [15]. Several other studies $[16,17]$ have shown $P$. aeruginosa is the second most common gram negative microorganism isolated from catheterized patient with UTI. The prevalence of $P$. aeruginosa in the current study was found to be $49.3 \%$. Compared to other similar studies (17\%) [16] the finding of this study is higher. It could be as a result of lack of consistent prescription policy and lack of reportable standard of care in our setup. In addition, duration of hospital stay and catheterization is also a major contributing factor $[18,19]$.

In this study higher proportion of females $(86.36 \%)$ were detected positive for $P$. aeruginosa than their male $(33.3 \%)$ counterparts while other studies reported higher prevalence of $P$. aeruginosa in males than in females [20, 21]. Nevertheless, it is comparable with the study from North Nigeria, where females found to be more infected by this bacterium [22]. Even though we did not control the study and thus cannot generate strong evidence from our data set physiological and anatomical factors might have played a role. The drier environment in the urethra, antimicrobial activity of prostate secretions and longer distance between the anus and urethra of males might delay or prevents the optimal growth of bacteria in males [23].

Another important factor in this study is age. The isolation rate was higher among old age greater than 45 years $(58.33 \%)$, and nearly $80.5 \%$ of the isolates were from age group greater than 35 years. In light of this study advancing age might be one of the predisposing factor. Furthermore there are studies from Nigeria that reported similar observation. Thus it is wise to give due attention to UTI when catheterizing older patients.

Increasing resistance to different antipseudomonal drugs particularly among hospital strains has been reported world-wide. This is a serious therapeutic problem in the management of disease due to these organisms. In our case we tested susceptibility profiles of $P$. aeruginosa to commonly used antimicrobial agents in the area. The isolate were susceptible to Ciprofloxacin and Norfloxacin. The Susceptibility of the isolates to Ciprofloxacin is very important for local consumption compared to other studies, whereby $40.5 \%$ [24], $50 \%$ [17] and $72.41 \%$ [25] susceptibility were observed, Ciprofloxacin should be given due attention and should be used when necessary as an alternative therapeutic agent for resistant isolates.

Aminoglycosides, especially gentamicin is a known frontline antibiotic in the treatment of bacterial infection by gram negative bacteria. However, emerging reports showed increased prevalence of resistance to these drugs. In this study, $13.88 \%$ isolates were observed resistant for this drug. It is lower than a study conducted in Nigeria [26], in which all isolates were resistant. The fact that we do share similar threat in our hospital impose another point of concern. 


\section{Conclusion}

The result shows higher prevalence of $P$. aeruginosa isolates among catheterized patients where the proportion of female patients detected positive for P. aeruginosa was higher than their male counterparts. All P. aeruginosa isolates were susceptible to Ciprofloxacin and Norfloxacin with some of the isolates shown resistance to Gentamicin. While the susceptibility of the isolates to the two fluoroquinolones is a good news for the prescribers their future rational prescription and use should be the main focus.

\section{Abbreviations}

AmpC: ampicillinase ( $\beta$-lactamase enzyme); AST: antimicrobial susceptibility testing; CAUTI: catheter associated urinary tract infections; CLSI: clinical laboratory standard institute; MDR: multidrug-resistant; UTI: urinary tract infections.

\section{Authors' contributions}

We declare here all authors have contributed (1) in conception and design, (2) acquisition of data (3) analysis and interpretation (4) drafting and revising the manuscript critically for intellectual content. All authors read and approved the final manuscript.

\section{Author details}

${ }^{1}$ Department of Pharmacy, College of Health Sciences, Jimma University, P. O. Box: 378, Jimma, Ethiopia. ${ }^{2}$ Department of Laboratory Sciences and Pathology, College of Health Sciences, Jimma University, Jimma, Ethiopia.

\section{Acknowledgements}

We acknowledge Jimma University for financial support and Study subjects for their willingness to participate in the study.

\section{Compliance with ethical guidelines}

\section{Competing interests}

The authors declare that they have no competing interests. There is no financial and non-financial competing interests to declare.

\section{Ethical considerations}

The study was ethically approved by Jimma University, College of Health Sciences-Ethical Review Board (CHS-ERB). Specimen collected only after verbal consent was obtained from study subjects.

Received: 18 April 2015 Accepted: 21 September 2015 Published online: 28 September 2015

\section{References}

1. Kenneth T. Online text book of bacteriology. 2005. http://textbookofbacteriology.net/ken_todar.html. Accessed 20 Jan 2015.

2. Cattle W. Infecting of the kidney and urinary tract. England: Oxford University Press; 1996. p. 1-26.

3. Mahmoud BA, Zahran AW, Hindawi RG, Labib ZA, Galal R. Prevalence of multidrug-resistant Pseudomonas aeruginosa in patients with nosocomial infections at a University Hospital in Egypt, with special reference to typing methods. J Virol Microbiol. 2013; 13.
4. Mesaros N, Nordmann P, Plesiat P, Roussel-Delvallez M, Van Eldere J, et al. Pseudomonas aeruginosa: resistance and therapeutics options in the turn of the new millennium. Clin Microbiol Infec. 2007;13(6):560-78.

5. Raja NS, Singh NN. Antimicrobial susceptibility pattern of clinical isolates of Pseudomonas aeruginosa in a tertiary care hospital. J Microbiol Immunol. 2007:40:45-9.

6. Bouza E, Garcia-Carrote F, Cercenado E, Marin M, Diaz M. Pseudomonas aeruginosa: a survey of resistance in 136 hospitals in Spain. Antimicrob Agents Ch. 1999:43:981-2.

7. Okon K, Agukwe P, Oladosu W, Balogun S, Uba A. Antibiotic resistance pattern of pseudomonas aeruginosa isolated from clinical specimens in a tertiary hospital in Northeastern Nigeria. internet J Microbiol. 2009; 8(2).

8. Al-Kabsi AM, Yusof MYBM, Sekaran SD. Antimicrobial resistance pattern of clinical isolates of Pseudomonas aeruginosa in the University of Malaya Medical Center, Malaysia. Afr J Microbiol Res. 2011;5(29):5266-72.

9. Wassef M, Mahallawy HE, Zafer MM, Ghaith D, Hamid RA. Lab based surveillance of multidrug resistant Pseudomonas aeruginosa in Cairo University Hospitals. Egypt J Microbiol Exp. 2015;. doi:10.15406/ jmen.2015.02.00039.

10. Gad GF, El-Domany RA, Zaki S, Ashour HM. Characterization of Pseudomonas aeruginosa isolated from clinical and environmental samples in Minia, Egypt: prevalence, antibiogram and resistance mechanisms. J Antimicrob Chemoth. 2007;60(5):1010-7.

11. Ullah F, Malik SA, Ahmed J. Antimicrobial susceptibility and ESBL prevalence in Pseudomonas aeruginosa isolated from burn patients in the north west of Pakistan. Burns. 2009;35(7):1020-5.

12. Vandepitte J, et al. Basisc laboratory procedures in clinical bacteriology. Geneva: WHO; 2003.

13. Cheesbrough M. District laboratory practice in tropical countries. 2nd ed. New York: Cambridge University Press; 2006.

14. CLSI. Performance standard for antimicrobial susceptibility test. 2012; 25(1): 100-15

15. Sayal P, Singh K, Devi P. Detection of bacterial biofilm in patients with indwelling urinary catheters. CIBTech J Microbiol. 2014;3(3):9-16.

16. Cetin B, et al. Epidemiology and etiology of catheter-related nosocomial infections in a Turkish hospital. Infez Med. 2005;13:152-9.

17. Roel Tongen, et al. Susceptibility Pattern of Bacterial Isolates from Catheterized Patients in a Referral Hospital. J Denta Med Sci. 2014;13(12):18-21.

18. Barre $\mathrm{S}$, et al. Antibiotic sensitivity of bacteria associated with community-acquired urinary tract infection in Britain. J Antimicrob Chemoth. 1999:44:359-65.

19. Grude N, Tveten Y, Kristiansen B. Urinary tract infections in Norway: bacterial etiology and susceptibility. A retrospective study of clinical isolates. Clin Microbiol Infec. 2001;7:543-7.

20. Jamshaid AK, et al. Prevalence and resistance patterns of Pseudomonas aeruginosa against various antibiotics. Pak J Pharm. 2008;21(3):311-5.

21. Rashid A, et al. Infections by Pseudomonas and antibiotic resistance pattern of the isolates from Dhaka Medical college Hospital. Bangladesh J Med Microbiol. 2007;1(02):48-51.

22. Jombo G. Multidrug resistant Pseudomonas aeruginosa in contemporary medical practice: findings from urinary isolates at a Nigerian University Teaching Hospital. Niger J Physiol Sci. 2008;23(1-2):105-9.

23. Hooton T. Pathogenesis of urinary tract infections: an update. J Antimicrob Chemoth. 2000;46(1):1-17.

24. Ahmed $\mathrm{S}$, et al. An emerging multi-drug resistant pathogen in a tertiary care centre in North Kerala. Annals Biol Res. 2012;3(6):2794-9.

25. Anil C, Shahid R. Antimicrobial susceptibility patterns of pseudomonas aeruginosa clinical isolates at a tertiary care hospital in kathmandu, Nepal. Asian J Pharm Clin Res. 2013;3:235-8.

26. Okon K. Antibiotic resistance pattern of Pseudomonas aeruginosa isolated from clinical specimens in a tertiary hospital in Northeastern Nigeria. J Microbiol. 2010;8(2):5-7. 